\title{
PHENIX Results and Plans on Charm and Dileptons
}

\section{Thomas K. Hemmick ${ }^{* \dagger}$}

Author Stony Brook University

E-mail: Thomas.Hemmickestonybrook.edu

\begin{abstract}
Although heavy quark and dilepton measurements share many aspects in their experimental determination, they probe rather different aspects of the collision. Leptons do not interact strongly and are thereby free to leave the medium during all stages of its evolution, hence earning the name "penetrating probes". Conversely, charm measurements (measured in PHENIX via semi-leptonic decay modes) are most remarkable in that they indicate a significant modification of the heavy quark spectrum through interactions with the medium. In this paper, we discuss a selection of intriguing results in both domains and the outlook for the future.
\end{abstract}

5th International Workshop on Critical Point and Onset of Deconfinement - CPOD 2009,

June 08 - 122009

Brookhaven National Laboratory, Long Island, New York, USA

*Speaker.

${ }^{\dagger}$ for the PHENIX Collaboration 


\section{Introduction}

Collisions at RHIC energy produce a new form of matter as evidenced by light quark energy

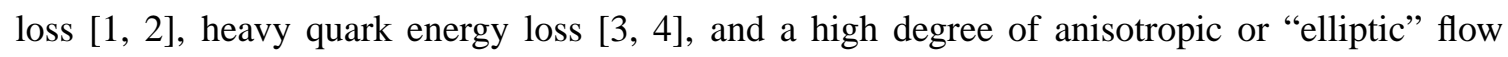
[4, 5, 6, 7]. The central questions at RHIC have moved beyond whether a new and interesting form of matter can be created and presently touch upon the detailed nature of this new matter, oft dubbed the strongly interacting Quark-Gluon Plasma or sQGP. Among these pressing questions are the initial state of the medium (Temperature and baryon chemical potential), its opacity, its fluid-like character $\left(\frac{\eta}{s}\right)$, and whether the phase diagram includes a critical point separating regions of $1^{\text {st }}$ and $2^{\text {nd }}$ order phase transitions.

Lepton studies provide particularly powerful tests of these most basic questions. Because leptons do not participate in the strong interaction, they can be released at all stages of the collision and thereby contain a component of direct thermal radiation from the initial state [8, 9] in the forms of virtual (subject of this paper) and real photons. Since the earliest observations of direct yields of virtual photon sources from the ISR [10] and UA1 measurements [11], a fascination with such measurements has developed. The discovery of low mass enhancement [12, 13], unique to heavy ion systems, has driven considerable theoretical [14, 15, 16] and experimental work at CERN [17, 18, 19], GSI [20], and most recently RHIC [21, 22].

Perhaps ironically, another application of lepton measurements is to measure the level of "perfection" of the initial fluid state at RHIC by deducing the viscosity to entropy density ratio $\left(\frac{\eta}{s}\right)$ from a simultaneous fit of heavy quark suppression and anisotropic flow [4]. Heavy quarks provide the most sensitive measure of $\frac{\eta}{s}$ by nature of their mass [23, 24, 25]. Comparison to several specific models [26, 27, 28] indicates a small value close to the theoretical lower bound [29].

\section{Dilepton Production}

Figure 11 shows a cartoon of the di-electron mass spectrum at full RHIC energy. For simplicity we discuss our results in three distinct mass regions. The High Mass Region (HRM) is above the J $\psi$ mass. The J/ $\psi$ and other heavy quarkonium states have long been a source of interest as these states, when they are rare, were initially expected to be suppressed by dissolution in a deconfined medium [30]. When not-so-rare, they may even exhibit enhancement via a new production mechanism: coalescence after dissolution even leading to a possible enhancement [31, 32]. The Intermediate Mass Region (IMR) lies between the $\phi$ and the $J / \psi$. At SPS energies this region exhibits an enhanced production [12, 17, 18, 33, 34, 35] that most recent studies have demonstrated does not come from heavy flavor, but from "prompt" decays [36]. As indicated in the cartoon, the higher energy at RHIC makes this region to be dominated by correlated open charm decays and is thereby a less sensitive regime for the identification and study of direct thermal radiation [37, 38, 39].

Finally is the Low Mass Region (LMR), bounded on the left by the peak of mesonic Dalitz decays and on the right by the $\rho / \omega$ region. This region is the most promising window for thermal radiation to out-shine hadronic and correlated heavy flavor and has indeed yielded a direct measurement of the thermal radiation from the plasma phase.

As is evident from Figure 1, a variety of hadronic di-electron sources compete with the measurements of the more interesting thermal radiation and correlated open heavy flavor leptons. 


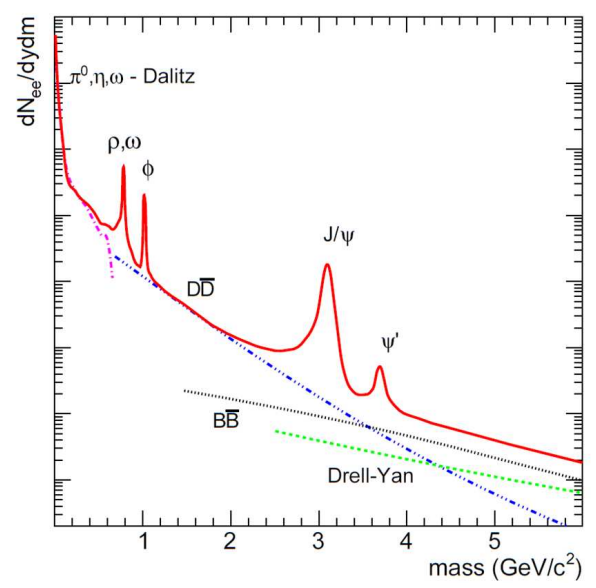

Figure 1: Schematic view of the dielectron spectrum. Continuum contributions will be considered in three distinct regions, separated by meson peaks. The High Mass Region (HMR) is above the J/ $\psi$; the Intermediate Mass Region (IMR) is between the $\phi$ and the $\mathrm{J} / \psi$; the Low Mass Region is between the $\pi / \eta$ Dalitz peak and the $\omega$.

PHENIX uses a home-grown event generator called EXODUS to model the hadronic component of these sources. The basic assumptions in EXODUS are spectral shapes taken from $m_{T}$ scaling of the measured pion yield, and thermal calculations of the integral yield. Where possible, data is used to adjust the thermal yield integrals [40, 41, 42, 43, 44, 45, 46, 47, 48, 49, 50, 51], however all such modifications are found to be minor due to the well-known success of thermal models at RHIC energies. The left panel of Figure 2 shows the remarkable agreement between data and the EXODUS model for a large variety of meson species. Heavy flavor decays are taken from the Pythia event generator, normalized when possible to PHENIX single electron data.

The right panel of Figure 2 shows the measured di-electron mass yield into the PHENIX acceptance as a function of mass for $\mathrm{p}+\mathrm{p}$ collisions at $\sqrt{s}=200 \mathrm{GeV}$ along with the EXODUS cocktail calculations. The colored band indicates the systematic error on the cocktail. The agreement of the mass spectrum to the cocktail is superb, indicating excellent understanding of the underlying production processes. The result of subtracting the hadronic sources away from the overall yield leaves only the correlated open heavy flavor component. The spectral shape of this yield is in good agreement with that of $1^{\text {st }}$ order QCD (Pythia). By allowing the normalization of charm and bottom decays to float freely, we can determine the cross sections for both these productions as $\sigma_{c \bar{c}}=544$ \pm 39 (stat) \pm 142 (syst) \pm 200 (model) $\mu \mathrm{b}$ and $\sigma_{b \bar{b}}=3.9 \pm 2.5$ (stat) ${ }_{-2}^{+3}$ (syst) $\mu \mathrm{b}$.

Figure 3 shows the comparison of the di-electron mass spectrum in $\mathrm{Au}+\mathrm{Au}$ collisions at $\sqrt{s}=200 \mathrm{GeV}$ to the same cocktail for Minimum Bias Collisions. Here the cocktail is seen to dramatically under predict the yield in the LMR. We will investigate this excess by slicing the mass spectrum into bands of transverse momentum. Panels (a) and (b) of Figure 7 show the data and cocktail in $p_{T}$ slices of $1 \mathrm{GeV} / \mathrm{c}$ and higher. Above $100 \mathrm{MeV} / \mathrm{c}^{2}$, an excess is noted in both $\mathrm{p}+\mathrm{p}$ and $\mathrm{Au}+\mathrm{Au}$ collisions. This excess may be attributed to direct virtual photon yield.

All processes that produce real photons also produce virtual photons that frequently materialize as $e^{+} e^{-}$pairs. The excess yield, as shown in the right panel of Figure 4 can be fit to a 

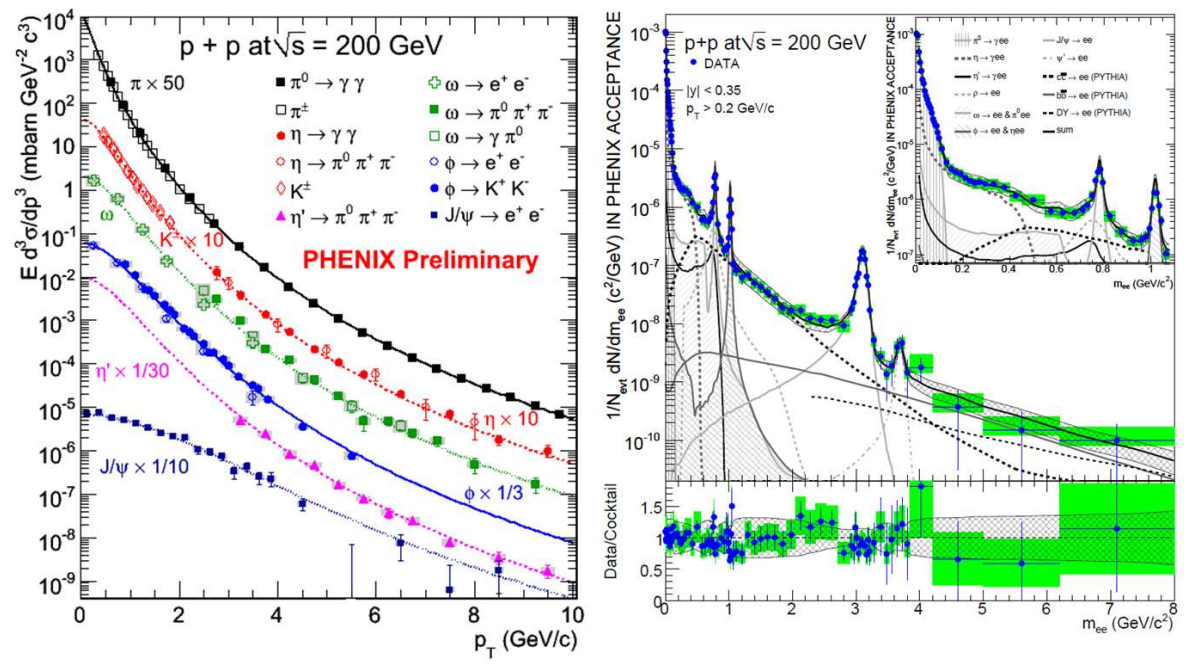

Figure 2: The left hand panel shows a comparison of measured meson yields with the "Cocktail" calculations. The "Cocktail" uses $m_{T}$ scaling from the pion spectrum normalized (where possible) by PHENIX data. All meson yields are well described and thereby yield accurate predictions for hadronic contributions to the di-electron spectrum. The right hand panel upper plot and inset show the measured di-electron yield in the PHENIX aperture as a function of pair mass along with curves indicating the hadronic and open charm/bottom cocktail. The lower plot is the ratio of data/cocktail demonstrating the precision with which the pp di-electron yields are understood.

two-component source containing both "cocktail processes" $\left(f_{c}(m)\right)$ and direct virtual photon yield $\left(f_{\text {dir }}(m)\right)$ as

$$
(1-r) f_{c}(m)+r f_{\text {dir }}(m)
$$

The $f_{\text {dir }}(m)$ form falls as $1 / \mathrm{m}$ in the limit $p_{T}>>m_{e e}$, valid for the region shown. The parameter " $\mathrm{r}$ ", as a function of transverse momentum, measures the fractional yield of direct virtual photons and thereby provides a bootstrap measurement of the overall direct photon yield [52]. .

The left panel of figure 5 shows the direct photon spectrum for $\mathrm{p}+\mathrm{p}$ collisions and $\mathrm{Au}+\mathrm{Au}$ collisions at various centralities along with $\mathrm{pQCD}$ calculations of this yield [53]. In the p+p system the yield is well explained by pQCD, but not so in the $\mathrm{Au}+\mathrm{Au}$ system. For $\mathrm{Au}+\mathrm{Au}$ there is a distinct strong component of direct photon yield at low transverse momentum. The latter component is well described for central collisions by a variety of calculations (right panel) [54, 53, 55, 56, 57, 58, 59] that assume formation of a hot system with initial temperature ranging from $T_{\text {init }}=300 \mathrm{MeV}$ at thermalization time $\tau_{0}=0.6 \mathrm{fm} / c$ to $T_{\text {init }}=600 \mathrm{MeV}$ at $\tau_{0}=0.15 \mathrm{fm} / c$ These experimental data are the most direct probe of the initial conditions of the RHIC fireball.

Figure 6 again shows $p_{T}$-sliced mass spectra for $\mathrm{p}+\mathrm{p}$ and $\mathrm{Au}+\mathrm{Au}$ collisions, however, this figure differs from Figure $\bigoplus$ in that slices continue to low transverse momentum. These data confirm that the majority of the LMR excess is generated with very low $p_{T}$. This excess is quantified in Figure 7. The left panel shows the $\mathrm{Au}+\mathrm{Au}$ data corrected to full azimuthal acceptance along with the cocktail calculations. This comparison indicates the presence of a two-component inverse slope 


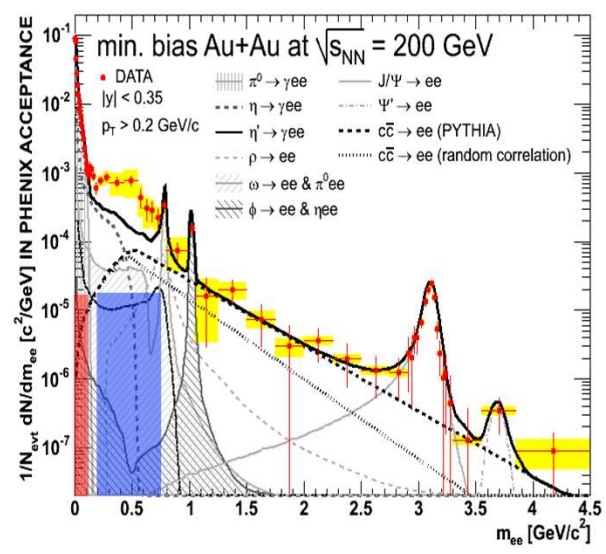

Figure 3: Di-electron yield into the PHENIX aperture for minimum bias collisions. A strong excess above cocktail sources is noted in the Low Mass Region (LMR).
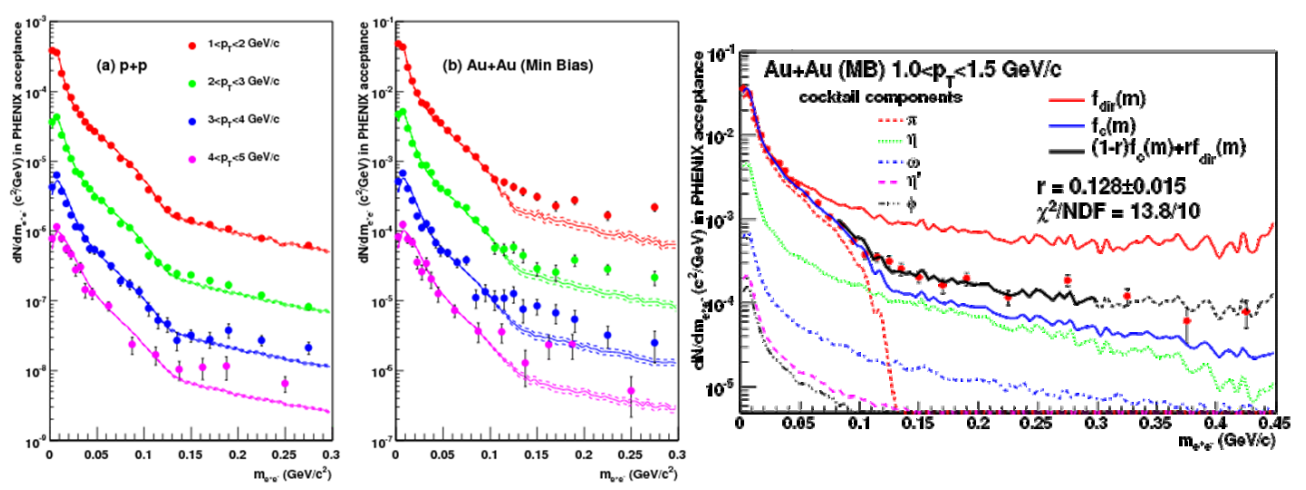

Figure 4: Di-electron mass spectra in various bands of transverse momentum. Cocktail overlays indicate an additional source of di-electrons above cocktail for masses between 100 amd $300 \mathrm{MeV} / c^{2}$. The parameter $r$ sets the relative fractional contributions of cocktail $\left(\left(f_{c}(m)\right)\right.$ and direct virtual photon yield $\left(f_{\text {dir }}(m)\right)$.

parameter. Indeed, local slope constants above and below $m_{T}=1 \mathrm{GeV}$ show distinctly different behaviors as shown in the right panel. The higher $m_{T}$ region has inverse slope constants similar to mesons of these masses and to SPS results [18]. Various model calculations (not shown) of Rapp and van Hees [37, 60, 61], Dusling and Zahed [62, 63, Cassing and Bratkovskaya [64, 65] all undershoot the full yield in the IMR, however do reproduce the yield above $m_{T}=1 \mathrm{GeV}$ reasonably well. None of these models explain the strong cold component observed in the data.

\section{Open Heavy Flavor}

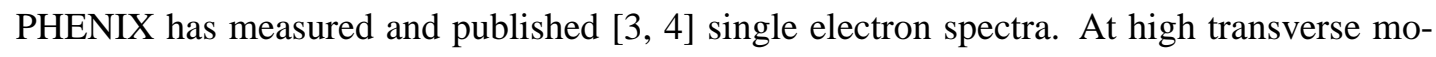
mentum, these spectra become dominated by electrons from semi-leptonic decays of heavy quarks. The published data include electrons from both closed and open heavy flavor decays. Recent pre- 

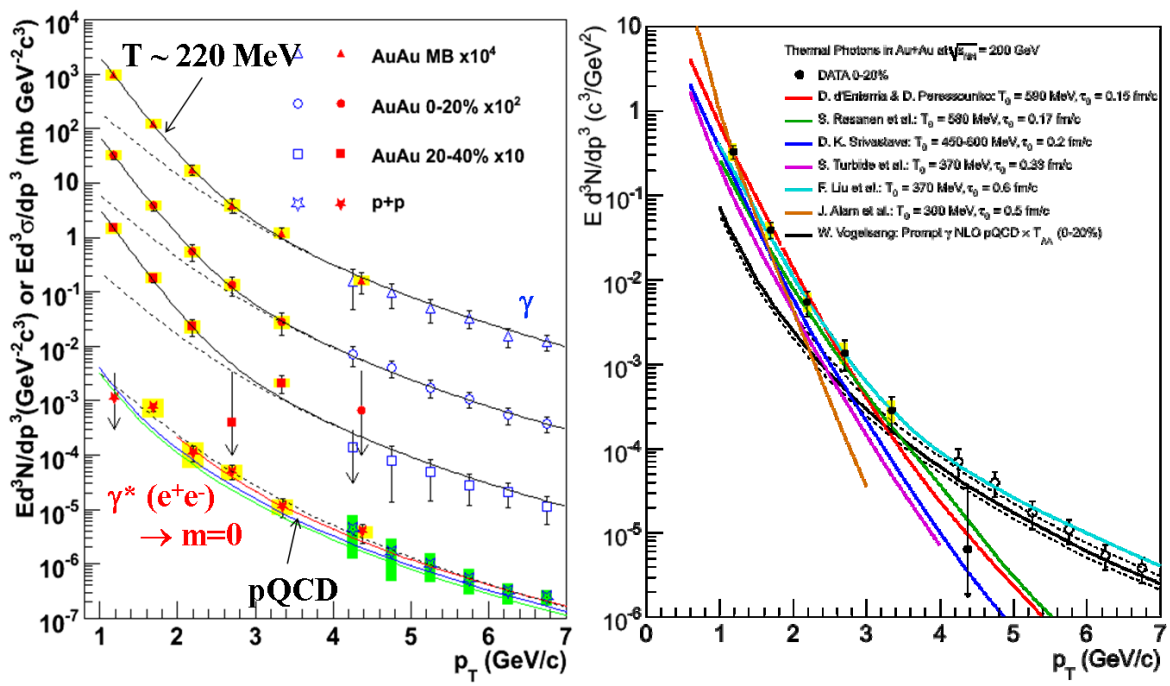

Figure 5: The left panel is an overlay of direct photon measurements via both direct virtual (red points) and direct real photons (blue points). The right panel is a comparison of PHENIX direct photon yield measurements with a variety of theoretical calculations with varying initial temperature and time assumptions.

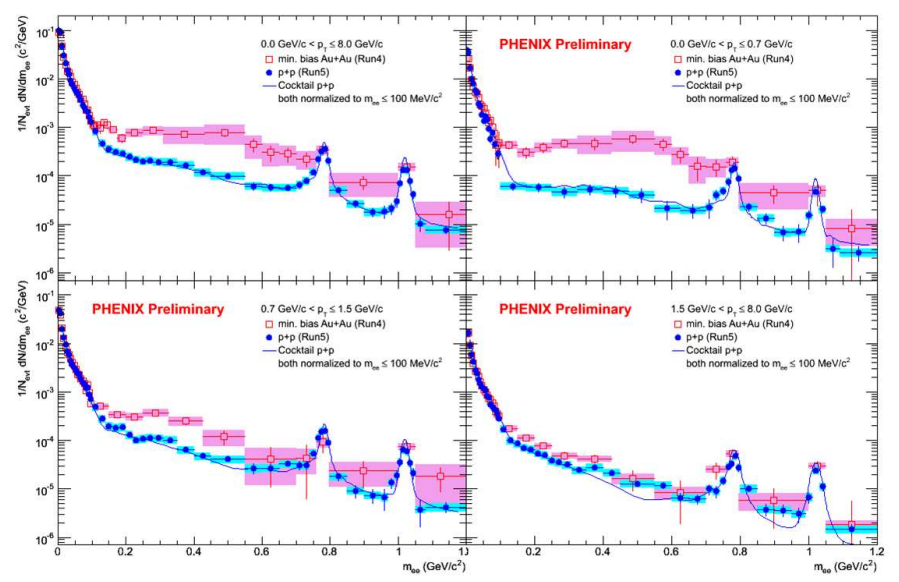

Figure 6: Transverse momentum sliced mass spectra for $\mathrm{p}+\mathrm{p}$ and $\mathrm{Au}+\mathrm{Au}$ colliding systems spanning $p_{T}$ down to the lowest measured. The bulk of the LMR excess comes primarily from the lowest transverse momentum range.

liminary results shown in Figure 8 not only improve the precision of these measurements, but also removed the contributions of single electrons from closed heavy flavor decays (charmonia).

These data indicate the remarkable result that heavy flavor quarks suffer strong spectral modification due the presensence of the medium and even pick up significant azimuthally anisotropic flow. Analysis in the context of several models [26, 27, 28], indicates an $\frac{\eta}{s}$ ratio within error equal to the lower bound. This result is all the more startling when one considers that recent PHENIX measurements [66] indicate that bottom quarks are significant contributors to the heavy quark elec- 

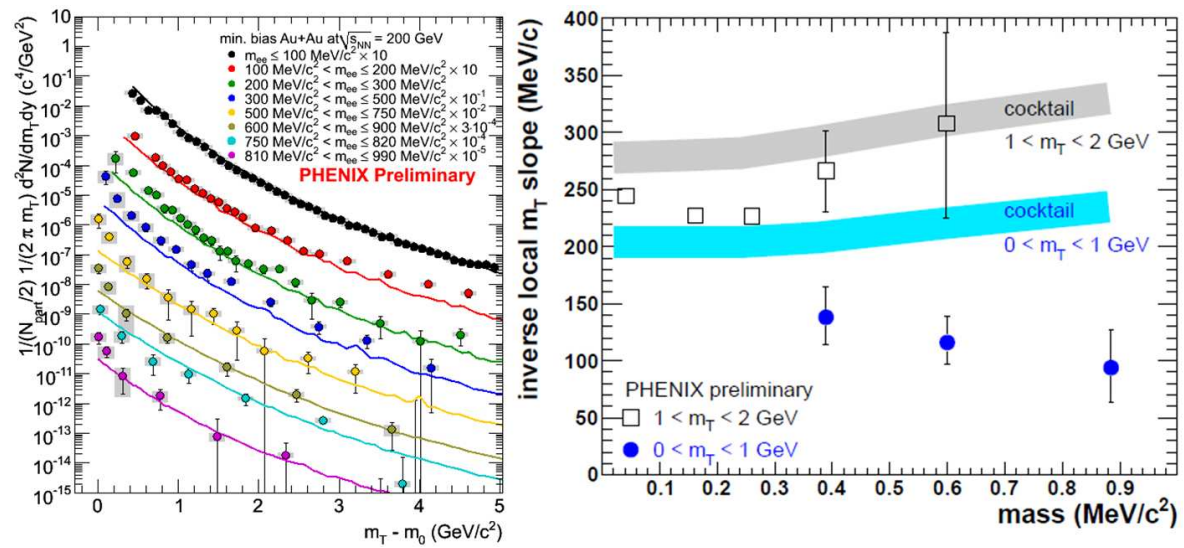

Figure 7: The left panel shows mass-sliced transverse momentum spectra. Dots are data and lines are cocktail. Data slices corresponding to the LMR region indicate that the bulk of the excess yield comes primarily from a steep component at low transverse momentum. The right panel shows inverse slope parameter as a function of mass in two different regions of $m_{T}$. Hollow points are high transverse mass that exhibit inverse slope parameters reminiscent of hadrons of similar mass. Solid points demonstrate the existence of a steep spectral component of net slope constant less than $100 \mathrm{MeV} / \mathrm{c}$.
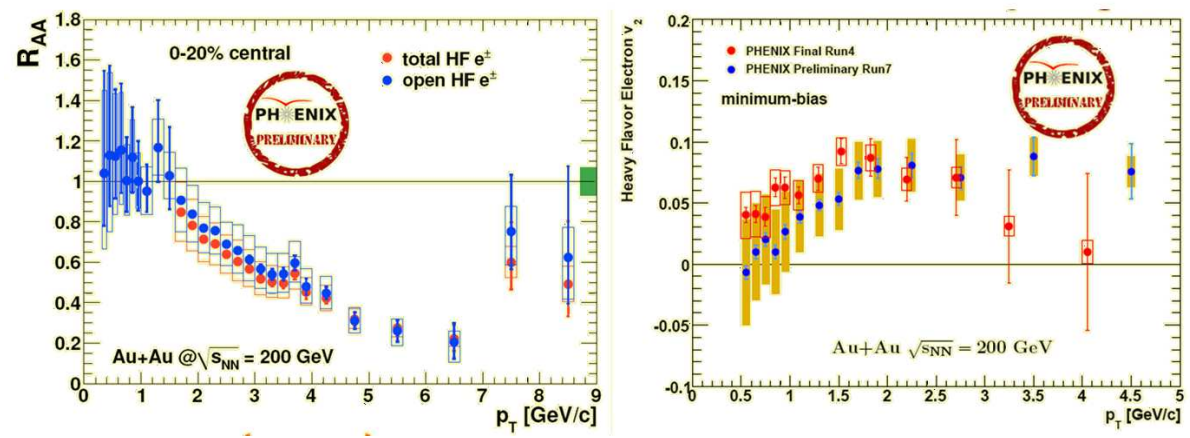

Figure 8: The left panel shows $R_{A A}$ of electrons from open heavy flavor decays indicating strong spectral modification well into the region likely dominated by bottom quarks. The right panel shows $v_{2}$ of electrons from open heavy flavor decays indicating that flow of heavy quarks persists to high transverse momentum.

trons beyond a $p_{T}$ of about $5 \mathrm{GeV} / \mathrm{c}$.

\section{Upgrade Plans}

The two topics of this paper are the subject of two upgrades. As shown in Figure 9 these two detectors vie for the same physical location, adjacent to the beam pipe. As such these two detector upgrades are set to be run sequentially.

The principal limitation to di-electron measurements in PHENIX stems from combinatorial background. With multiple $e^{+} e^{-}$pairs produced in a single collision, many "false pairings" $\left(e^{+}\right.$ from one decay paired with an $e^{-}$from another decay) occur. This background is so severe for full energy $\mathrm{Au}+\mathrm{Au}$ collisions that the signal constitutes less than 1 part in 100 for certain masses. 


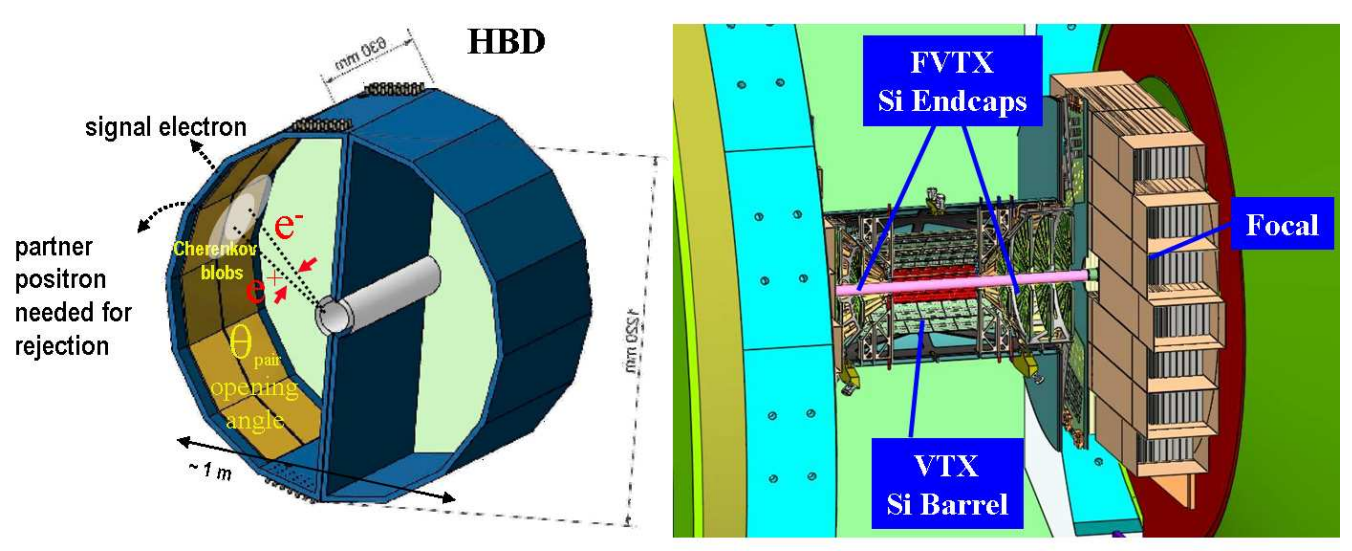

Figure 9: Two detector upgrades vie for space near the collision point. The left panel shows a mechanical drawing of the PHENIX Hadron-Blind detector. The right panel shows the VTX, FVTX, and FOCAL upgrades.

Fortunately, false pairings can be controlled experimentally. The most copious physical source of $e^{+} e^{-}$pairs is the Dalitz decay of the $\pi^{0}$. The second most copious source is conversion of real photons to $e^{+} e^{-}$in the material of the PHENIX detector. Both of these sources produce pairs with small invariant mass and small opening angle. The PHENIX HBD seeks to tag electrons with close angular partners by detecting Cherenkov light with the PHENIX magnetic field canceled in the central region. Calculations show that the HBD will improve the effective signal by more than an order of magnitude if a single electron response of more than 18 photo-electrons can be achieved.

The Hadron-Blind Detector is a unique device [67, 68, 69, that uses a triple stack of Gas Electron Multipliers (GEMs) to detect Cherenkov light. The first GEM in the stack is coated with an evaporated layer of CsI and serves as the photo-cathode. This GEM is preceded by a mesh that directs ionization electrons (from hadrons) away from the avalanche. However the strong electric field in the vicinity of the GEM holes directs nearly all photo-electrons to avalanche. Shown in Figure 10 are online measured responses of the HBD to isolated single electrons and overlapping close angle pairs. These plots indicate that the HBD is functioning well. We expect to make vastly improved di-electron measurements in Run-10.

After Run-10, the HBD will be removed and replaced by the vertex detectors. The VTX pixel \& strip detector will be installed for Run-11 and the FVTX will also become available in Run-12. The VTX will allow direct identification of heavy flavor decays via their source being a displaced vertex. The innermost 2 layers of the VTX consist of $50 \mu \mathrm{m}$ by $425 \mu \mathrm{m}$ pixels at radii of $2.5 \mathrm{~cm}$ and $5 \mathrm{~cm}$. The outer two layers are "strip-pixel" devices with an $80 \mu \mathrm{m}$ by $1000 \mu \mathrm{m}$ pitch. The device covers $-1.2<\eta<1.2$ and is nearly complete in azimuth.

Figure 11 shows the anticipated precision for $R_{A A}$ and $v_{2}$ measurements with this device in a run at RHIC-II luminosity. This upgrade will usher in a new era of heavy flavor physics at RHIC by distinguishing charm from bottom in all measurements. 


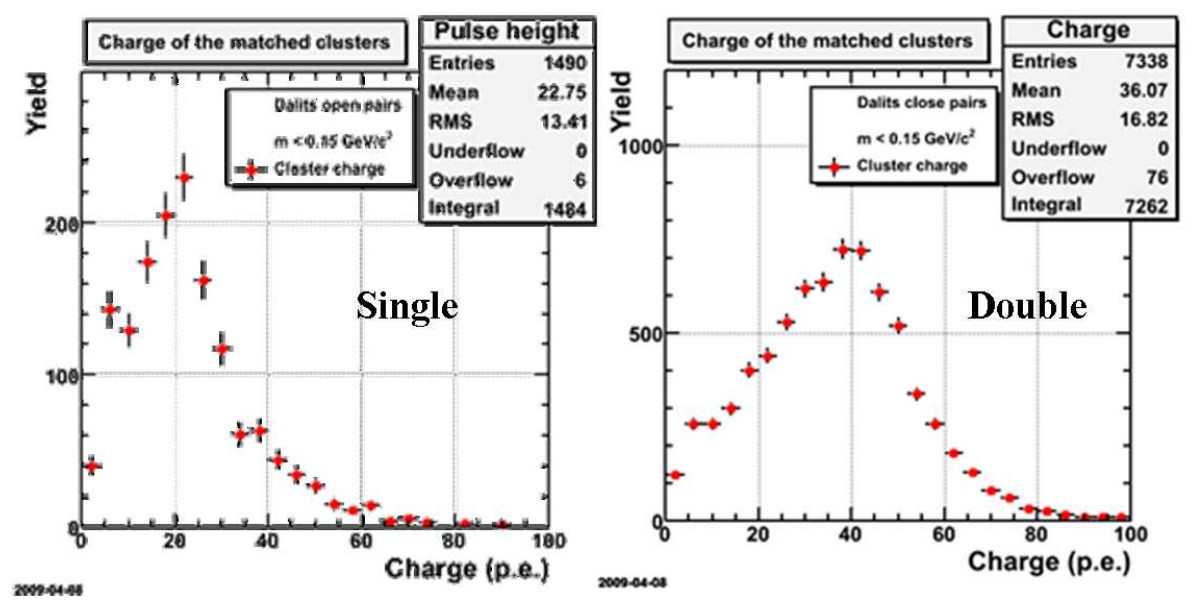

Figure 10: The left panel shows the measured HBD response to isolated single electrons. The right panel shows the response to overlapping or "double" electrons.

PHENIX VXT $\sim 2 \mathbf{n b}^{-1}$
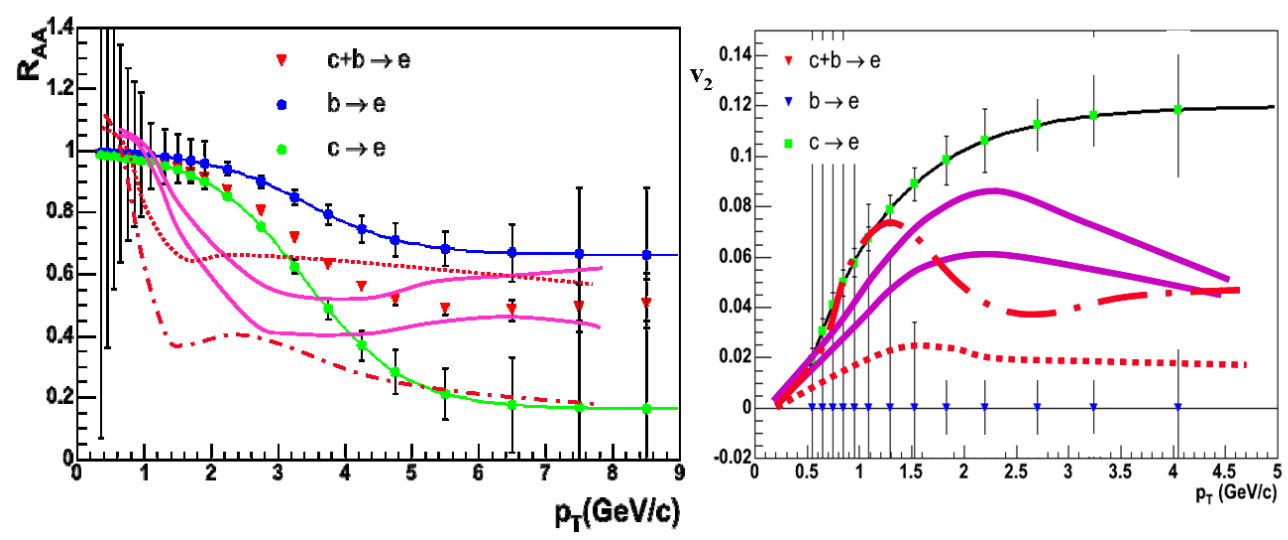

Figure 11: The left panel shows the expected $R_{A A}$ precision from the VTX detector for one run under RHIC-II Luminosity. The right panel is the same for $v_{2}$. In both cases, the VTX detector allows for distinct measurements of charm and bottom quarks.

\section{References}

[1] K. Adcox et al., Phys. Rev. Lett. 88, 022301 (2002).

[2] S.S. Adler et al., Phys. Rev. Lett. 96, 202301 (2006).

[3] S.S. Adler et al., Phys. Rev. Lett. 96, 032301 (2006).

[4] A. Adare et al., Phys. Rev. Lett. 98, 252002 (2006).

[5] S.S. Adler et al., Phys. Rev. Lett. 91, 182301 (2003).

[6] A. Adare et al., Phys. Rev. Lett. 98, 162301 (2007).

[7] S. Afanasiev et al., Phys. Rev. Lett. 99, 052301 (2007). 
[8] P. Stankus, Ann. Rev. Nucl. Part. Sci. 55, 517 (2005).

[9] R. Rapp and E. Shuryak, Phys. Lett. B473, 13 (2000).

[10] J. H. Cobb et al., Phys. Lett. B78, 519 (1978).

[11] C. Albajar et al., Phys. Lett. B209, 397 (1988).

[12] M. Masera, Nucl. Phys. A590, 103c (1995).

[13] G. Agakichiev et al., Phys. Rev. Lett. 75, 1272 (1995).

[14] G.E. Brown and M. Rho, Phys. Rev. Lett. 66, 2720 (1991).

[15] R. Rapp, J.Wambach, Adv. Nucl. Phy. 25, 1 (2000) and references therein.

[16] W. Cassing and E. Bratkovskaya, Phys. Rep. 308, 65 (1999) and references therein.

[17] R. Arnaldi et al., Phys. Rev. Lett. 96, 162302 (2006).

[18] R. Arnaldi et al., Phys. Rev. Lett. 100, 022302 (2008).

[19] G. Agakichiev et al., Eur. Phy. J. C41 475 (2005).

[20] G. Agakichiev et al., Phys. Rev. Lett. 98, 052302 (2007).

[21] S. Afanasiev et al., submitted to Phys. Rev. Lett. [arXiv:nucl-ex/07063034].

[22] A. Adare et al., Phys. Lett. B670, 313 (2009).

[23] E. Shuryak, Prog. Part. Nucl. Phys. 53, 273 (2004).

[24] M. Gyulassy, L. McLerran, Nucl. Phys. A750, 30 (2005).

[25] P. F. Kolb, U. W. Heinz, nucl-th/0305084.

[26] N. Armesto, et al., Phys. Lett. B637, 362 (2006).

[27] H. van Hees, V. Greco, R. Rapp, Phys. Rev. C73, 034913 (2006) and private communication.

[28] G. D. Moore, D. Teaney, Phys. Rev. C71, 064904 (2005) and private communication.

[29] P. Kovtun, D. T. Son, A. O. Starinets, Phys. Rev. Lett. 94, 111601 (2005).

[30] T. Matsui and H. Satz, Phys. Lett. B178, 416 (1986).

[31] P. Braun Munzinger and J. Stachel, Phys. Lett. B490, 196 (2000) and P. Braun-Munzinger, K. Redlich and J. Stachel, "Quark Gluon Plasma. Vol 3”, World Scientific, Singapore, nucl-th/0304013 (2003).

[32] R.L. Thews, M. Schroedter, J. Rafelski, Phys. Rev. C63, 054905 (2001).

[33] A.L.S. Angelis et al., Eur. Phy. J. C13, 433 (2000).

[34] M.C. Abreu et al., Phys. Lett. B368 230(1996).

[35] M.C. Abreu et al., Eur. Phy. J. C14, 443 (2000).

[36] R. Arnaldi et al., Eur. Phy. J. C61, 711 (2009).

[37] R. Rapp, Phys. Rev. C63, 054907 (2001).

[38] K. Gallmeister, B. Kaempfer and O.P. Pavlenko Phys. Rev. C57, 3276 (1998) and B. Kaempfer, O.P. Pavlenko and K. Gallmeister, Phys. Lett. B419, 412 (1998).

[39] E.V. Shuryak Phys. Rev. C55, 961 (1997) and C.M. Hung, E.V. Shuryak, Phys. Rev. C56, 453 (1997). 
[40] A. Adare et al., Phys. Rev. D76, 051106 (2007).

[41] S.S. Adler et al., Phys. Rev. C74, 024904 (2006).

[42] S.S. Adler et al., Phys. Rev. C75, 024909 (2007).

[43] S.S. Adler et al., Phys. Rev. C75, 051902 (2007).

[44] Y. Riabov et al., J. Phys G34, No.8, S925 (2007).

[45] A. Adare et al., Phys. Rev. Lett. 98, 232002 (2007).

[46] S.S. Adler et al., Phys. Rev. Lett. 91, 072301 (2003).

[47] S.S. Adler et al., Phys. Rev. C69, 034909 (2004).

[48] S.S. Adler et al., Phys. Rev. C72, 014903 (2005).

[49] A. Adare et al., Phys. Rev. Lett. 98, 232301 (2007).

[50] V. Ryabov, Nucl. Phys. A827, 1-4, 395c (2009).

[51] A. Milov, Munich 2007, Deep-inelastic scattering, 731 [arXiv:nucl-ex/0707.1258] (2007) and Y. Nakamiya, J. Phys GNucl. Part. Phys. 35, 104158 (2008).

[52] A. Adare, et al., submitted to Phys. Rev. Lett. [arXiv:nucl-ex/0804.4168].

[53] L. E. Gordon and W. Vogelsang, Phys. Rev. D48, 3136 (1993) and W. Vogelsang, private communication (2008).

[54] D. d'Enterria and D. Peressounko, Eur. Phy. J. C46, 451 (2006).

[55] S. Turbide R. Rapp, and C. Gale, Phys. Rev. C69, 014903 (2004).

[56] P. Huovinen, P. V. Ruuskanen, and S. S. Rasanen, Phys. Lett. B535, 109 (2002).

[57] D. K. Srivastava and B. Sinha, Phys. Rev. C64, 034902 (2001).

[58] J.-e. Alam et al., Phys. Rev. C63, 021901 (2001).

[59] F.-M. Liu et al., Phys. Rev. C79, 014905 (2009).

[60] R. Rapp, nucl-th/0204003; W. Liu, R. Rapp, Nucl. Phys. A796, 101 (2007).

[61] R. Rapp, private communication. The numerical table of the double differential yield of lepton pair based on the same theoretical model of [70] is provided by Rapp.

[62] K. Dusling and I. Zahed, Nucl. Phys. A825, 212 (2009) and K. Dusling PhD thesis, Stony Brook University (2008).

[63] K. Dusling, private communication. The numerical table of the double differential yield of lepton pair based on the same theoretical model of [62] is provided by Dusling.

[64] E.L. Bratkovskaya, W. Cassing, O. Linnyk, Phys. Lett. B670, 428 (2009).

[65] E.L. Bratkovskaya, private communication. The numerical table of the double differential yield of lepton pair based on the same theoretical model of [64] is provided by Bratkovskaya.

[66] A. Adare et al., Phys. Rev. Lett. , 103, 082002 (2009).

[67] A. Kozlov et al., Nucl. Instrum. Meth., A523 345-354 (2004).

[68] Z. Fraenkel et al., Nucl. Instrum. Meth., A546, 466-480 (2005).

[69] B. Azmoun et al.IEEE Transactions 57:3 1544-1549 (2009).

[70] H. van Hees and R. Rapp, Nucl. Phys. A806, 339 (2008). 